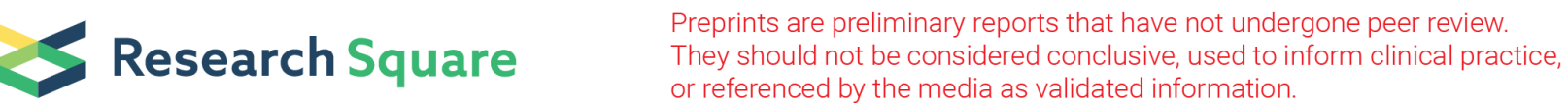

\section{Age-specific associations of blood pressure variability with cardiovascular disease and mortality: 10-year Diabetes cohort study}

Eric Yuk Fai WAN ( $\square$ yfwan@hku.hk)

University of Hong Kong https://orcid.org/0000-0002-6275-1147

Esther Yee Tak Yu

University of Hong Kong

Weng Yee Chin

University of Hong Kong

Jessica K. Barrett

University of Cambridge

lan Chi Kei Wong

University of Hong Kong

Esther Wai Yin Chan

University of Hong Kong

Celine Sze Ling Chui

University of Hong Kong

Shiqi Chen

University of Hong Kong

Cindy Lo Kuen Lam

University of Hong Kong

Original investigation

Keywords: Diabetes; Variability; Blood pressure; Cardiovascular Disease; Mortality

Posted Date: March 10th, 2020

DOl: https://doi.org/10.21203/rs.3.rs-16513/v1

License: (c) (i) This work is licensed under a Creative Commons Attribution 4.0 International License.

Read Full License 


\section{Abstract}

Background: This study evaluated the age-specific association of systolic blood pressure variability with cardiovascular disease and mortality in Type-2 diabetic patients. The detrimental effects of increased systolic blood pressure variability on cardiovascular disease and mortality risk in diabetic patients remains unclear. Methods: A retrospective cohort study investigated 155,982 diabetic patients aged from 45 to 84 years old without prior history of cardiovascular disease at baseline from 2008 to 2010). systolic blood pressure variability was estimated using systolic blood pressure standard deviation from mixed effects model to reduce regression dilution bias. Age-specific associations (45-54; 55-64; 65-74; 75-84 years) between systolic blood pressure variability, cardiovascular disease and mortality risk were assessed by Cox regression adjusted for patient characteristics with subgroups stratified by subject baseline characteristics. Results: After a median follow-up of 9.7 years (16.4 million person-years), 49,816 events (including 34,039 events with and 29,211 mortalities) were identified. Elevated and independent systolic blood pressure variability was positively and log-linearly associated with higher risk on cardiovascular disease and mortality among all age groups, without evidence of a specific threshold. The cardiovascular disease and mortality risk per $5 \mathrm{mmHg}$ increase in systolic blood pressure variability within 45-54 years age group is over three times higher than the 70-79 years age group [Hazard Ratio: 1.66 (1.49, $1.85)$ vs. Hazard Ratio: $1.19(1.15,1.23)]$. The significant associations remained consistent among all subgroups. Patients with younger age, lower systolic blood pressure and comorbidity with more types of anti-hypertensive prescription drug users had higher hazard ratios. Conclusions: The findings suggest that systolic blood pressure variability was strongly associated with cardiovascular disease and mortality risk without evidence of a specific threshold in diabetic population. In addition to optimize blood pressure control, the systolic blood pressure variability particularly for younger patients should be monitored and evaluated in routine practice.

\section{Background}

Diabetes Mellitus (DM) is one of the most predominant chronic diseases, affecting 425 million people worldwide, and the number is estimated to rise to 629 million by year 2045 (1). With a high prevalence of $8.8 \%, \mathrm{DM}$ is a source of great burden for global healthcare due to its high incident complications (2). Compared to patients without DM, diabetic patients have at a 2-3 fold higher risk of cardiovascular disease (CVD), which is a key factor contributing to mortality and disability, leading to 5 million deaths in 2015 (3). The benefits of blood pressure control, in particular systolic blood pressure (SBP), in preventing CVD is well studied in diabetic populations (4-7), and thus several international guidelines on diabetes management have established the SBP target $(8,9)$. As a consequence, most clinicians do not pay much attention to the variability in SBP as long as the SBP is controlled to target. However, the SBP variability has drawn particular attention in diabetic patients.

A recent systematic review found a relationship between long-term SBP variability and higher risks for CVD and mortality in diabetic population (10). However, only six and five studies included in the review for the CVD and mortality outcome, respectively. A recent a study conducted in a Swedish diabetic 
population managed in primary care settings showed that SBP variability was associated with all-cause mortality for patients without CVD and BP lowering medications only but not for other diabetic patients (11). However, most of these studies performed to date have only included a small number of patients and outcome events, had short follow-up periods, and have not made adjustments for mean SBP over time, or had used post-baseline measurements to determine SBP variability, post-baseline measurements, which may have informative censoring or immortal time bias $(12,13)$. Only our previous study and another post-hoc study of Action in Diabetes and Vascular Disease: Preterax and Diamicron Modified Release Controlled Evaluation (ADVANCE) randomized controlled trial (RCT) obtained the SBP variability based on pre-baseline measurements $(14,15)$. Nevertheless, the follow-up period in our previous study was only 3.3 years that may not able to evaluate long term (e.g. 10 years) effect of SBP variability on the outcome events (14). Meanwhile, the longer follow-up period of median 7.6 years in study from ADVANCE RCT limited their strict inclusion criteria (e.g. with high risk of vascular events) (15), and thus results may not be generalizable given the heterogeneity in the general diabetic population. More importantly, the measures of SBP variability are estimated on a person-by-person basis and thus subject to measurement error, which causes regression dilution bias in the estimated association between SBP variability and event outcomes (16). In addition, the association between SBP and CVD may vary in different age groups $(12,17,18)$, but there is no available study evaluating the age-specific effects of SBP variability on CVD and mortality amongst diabetic patients.

This cohort study aimed to update our previous work using longer follow-up period (10-year) and the adjustment for regression dilution bias to evaluate the age-specific association between SBP variability with CVD and mortality in patients with type 2 DM managed in Hong Kong primary care settings (14). The pattern of variability among patients with different baseline characteristics were also explored. A more comprehensive knowledge of the effect of SBP variability can help enable clinicians better risk stratify and identify patients at higher risks in advance.

\section{Methods}

\section{Study Design}

This is retrospective cohort study, which obtained the data from prospectively population clinical database from the Hong Kong Hospital Authority (HA). Patients were included if they were aged 45-84 years, with a clinical diagnosis of type $2 \mathrm{DM}$, with no prior diagnosis of CVD at baseline, and managed in public primary care clinics of the Hong Kong HA from 1 January 2008 to 31 December 2010. The HA is the chief regulatory institution for all 42 public-sector hospitals, 47 specialist outpatient clinics, and 73 primary care clinics in Hong Kong. Over $90 \%$ of the local population with chronic disease receive medical treatments through the HA(19). Diagnosis of type $2 \mathrm{DM}$ was made by HA clinic doctors and recorded using the International Classification of Primary Care-2 (ICPC-2) code of T90. All confidential data, including patients' characteristics and event outcomes, are kept in the electronic health database of the HA's clinical management system (CMS). The validity and coding accuracy of the CMS database has been well established and adopted in several previous high-quality population-based epidemiological 
studies $(14,20,21)$. Clinicians and related healthcare professionals receive training in using the CMS to record a variety of the clinical information and patient demographics simultaneously during the doctor consultation, including patients' diagnoses, prescriptions, laboratory tests and results, emergency department visits, hospitalizations, and specialist and primary care outpatient clinic visits. The timeline of the measurements of SBP and outcome determination is presented in Figure S1. Most diabetic patients managed in public primary care clinics are typically followed-up once per quarter. Hence, a first SBP reading with 8 repeated readings at every 3 months until the end of 2-year visit (total 9 occasions) was adopted to identify the SBP variability. Each patient was continuously tracked until the incident date of outcome events, death, or the last follow-up visit until 31 December 2017, whichever occurred first.

\section{Outcome Measures}

The primary outcome was the incidence of the composite of CVD and all-cause mortality. The secondary outcomes consisted of individual CVD, the subtype of coronary heart disease (CHD), stroke, heart failure, all-cause mortality, CVD mortality, and non-CVD mortality. The outcome events was defined using the ICPC-2 or the International Classification of Diseases, Ninth Edition, Clinical Modification (ICD-9-CM), the officially admitted diagnostic codes. Hong Kong Government Death Registry provided the mortality reports retrieved from their internal population data, where CVD-related mortality was classified as the death with a history of CVD or the main cause of death record by the International Classification of Diseases, Tenth Edition codes of I20-I25, I50, and I60-169. In previous studies, these codes have revealed a high coding accuracy in diagnosing myocardial infarction and stroke with positive predictive values of 85.4\% (95\% confidence interval (Cl) 78.8-90.6\%) and 91.1\% (83.2-96.1\%), respectively (21). Table S1 also summarized all the definitions for individual event.

\section{Clinical BP Measurements}

In the process of obtaining and documenting SBP readings in patients during the consultations, all clinics strictly followed a standardized guideline [27]. Patients are instructed to maintain a seated position for 5 minutes without any distraction before the start of measurements of SBP, and to have at least 1 minute rest between every two measurements. Nurses or trained patient care assistants used a standardized automated sphygmomanometer (UA-853, Tokyo, Japan; EDAN M3A, Shenzhen, China, or other equivalent measurements). If the difference between the two readings exceeded $5 \mathrm{mmHg}$, extra measurements would be taken. The average of these SBP readings was calculated and recorded as the SBP measurement result.

\section{Usual SBP and SBP variability Measurements}

To minimize regression dilution bias, usual SBP and SBP variability measures were obtained using a mixed effects model, which allows the within-individual variability to differ between individuals. Longitudinal trajectories were modelled by including a slope term as both a fixed and random effect. Bayesian Markov chain Monte Carlo was used to fit the mixed effects model. The usual SBP and SBP variability were estimated by the posterior mean of the random intercept and the residual standard deviation, respectively. Details of the calculation of usual SBP and SBP variability measures have been 
described in the Supplementary document and elsewhere in the literature (16). Patients with fewer than four SBP records were excluded from this study to increase the precision of the usual SBP and SBP variability estimates. The average total number of SBP measurements was 4.5 (SD: 1.3). The analysis was implemented using JAGS Version 3.4.0 and the R2jags package in $\mathrm{R}(22,23)$.

\section{Baseline characteristics}

Baseline covariates encompassed gender, age, smoking status, body mass index (BMI), SBP, diastolic blood pressure, Haemoglobin A1c (HbA1c), low-density lipoprotein-cholesterol (LDL-C), estimated glomerular filtration rate (eGFR), the Charlson's comorbidity index $(24,25)$, the use of anti-hypertensive drug (e.g. angiotensin converting enzyme inhibitor or angiotensin receptor blocker (ACEI/ARB), $\beta$-blocker, calcium channel blocker (CCB), diuretics and others (hydralazine, methyldopa, and prazosin)), oral antidiabetic drugs, insulin, and lipid-lowering agents. The eGFR was computed in accordance with the creatinine level from blood testing along with the abbreviated Modification of Diet in Renal Disease Study formula recalibrated for Chinese (eGFR in $\mathrm{ml} / \mathrm{min} / 1.73 \mathrm{~m}^{2}=186 \times[$ (serum creatinine in $\mu \mathrm{mol} / \mathrm{L}) \times$ $0.011]-1.154 \times(\mathrm{age})^{-0.203} \times(0.742$ if female $) \times 1.233$, where 1.233 is the adjusted coefficient for local population (26). All laboratory assays were performed in accredited laboratories by the College of American Pathologists, the Hong Kong Accreditation Service, or the National Association of Testing Authorities, Australia.

\section{Data Analysis}

Multiple imputation was used to impute missing data for all baseline characteristics apart from blood pressure. The chained equation method was used to impute each missing value five times, adjusted for all baseline covariates and outcomes. The same analysis method was adopted for each of the five imputed datasets, and the results were pooled using Rubin's rule (27).

All patients were allocated into one of five groups defined by their SBP variability $(<10 \mathrm{mmHg}, 10-$ $11.9 \mathrm{mmHg}, 12-13.9 \mathrm{mmHg}, 14-15.9 \mathrm{mmHg}, \geq 16 \mathrm{mmHg}$ ). After multiple imputation, descriptive statistics were displayed for integration of the baseline characteristics for each group. The cumulative incidence and incidence rate of CVD, mortality, and their composite events were reported and the confidence interval (Cl) of the incidence rate was estimated using the Poisson distribution. Age-specific associations (45-54, 55-64, 65-74 and 75-84 years) between SBP variability and the risk of an event were estimated by multivariable Cox proportional hazards regressions adjusted for all baseline covariates and usual SBP. An additional term was fitted to allow the HR within each age group to be assessed as the geometric mean of the HRs in the first and second half of that decade (28). Thus, the hazard ratios comparing event risk between different SBP variability groups were not assumed to be similar for different age decades. The $95 \% \mathrm{Cl}$ of the hazard ratios were estimated using the floating absolute risk, without the requirement of selecting a reference group for displaying the standard error (29). Details of the above methods have previously been described in the literature $(28,29)$, and they have been widely adopted in several large epidemiological studies (30-32). To confirm the shape of the association, restricted cubic splines with three knots in Cox models were used for SBP variability, treated as a continuous variable (33). To 
enhance the robustness of the results, other variability measurements such as coefficient of variation and variability independent of mean rather than SD were used. Five sensitivity analyses were also conducted. Firstly, Rosner's regression method was used to calculate the regression dilution ratio (34). The details of this approach are described in the Supplementary document. Secondly, a complete case analysis was performed. Thirdly, patients were excluded if they attended less than 1-year follow-up. Fourthly, the analyses were implemented for patients with $\geq 2$ and $\geq 7$ rather than $\geq 4$ SBP measurements. Fifthly, the usage of aspirin

To further explore the effect of SBP variability on the outcomes for patients with different characteristics, subgroup analyses were performed which were stratified by gender (male; female), age at risk (45-54, 55-64, 65-74, 75-84 years), smoking status (non-smoker, smoker), BMI ( $<25 ; \geq 25 \mathrm{~kg} / \mathrm{m}^{2}$ ), baseline SBP $(<130,130-139,140-149, \geq 150 \mathrm{mmHg})$, usual SBP $(<130,130-139,140-150, \geq 150 \mathrm{mmHg})$, LDL-C $(<2.6, \geq 2.6 \mathrm{mmol} / \mathrm{L}), \mathrm{HbA} 1 \mathrm{c}(<7, \geq 7 \% \mathrm{mmol} / \mathrm{mol})$, eGFR $\left(<90, \geq 90 \mathrm{ml} / \mathrm{min} / 1.73 \mathrm{~m}^{2}\right)$, Charlson's Index $(<4, \geq 4)$, the prescription of number of type of anti-hypertensive drugs $(0,1,2,3)$, and different antihypertensive drugs.

All significance tests were two-tailed and those with a p-value less than 0.05 were considered statistically significant. The statistical analysis was executed in Stata Version 13.0.

\section{Results}

A cohort of 155,982 patients with Type 2 diabetes satisfied the inclusion and exclusion criteria and were included in the analyses. Table S2 illustrates that the data completion rate for all baseline characteristics was higher than 84.3\%, except for eGFR (77.1\%), BMI (64.6\%), and LDL-C (63.6\%). In Table 1, patient's characteristics are summarized in each SBP variability group at baseline after multiple imputations. Overall, the mean age was 63.9 years (SD: 10.0) and males account for $45.2 \%$. Usual SBP mean and SBP variability were $137.1 \mathrm{mmHg}(\mathrm{SD}: 10.7)$ and $12.7 \mathrm{mmHg}$ (SD: 2.3), respectively. 
Table 1

Descriptive statistics for baseline characteristics among patients stratified by SBP variability

\begin{tabular}{|c|c|c|c|c|c|c|}
\hline & \multicolumn{5}{|c|}{ SBP variability } & \multirow{2}{*}{$\begin{array}{l}\text { Overall } \\
(\mathrm{N}= \\
155,982)\end{array}$} \\
\hline & $\begin{array}{l}< \\
10 \mathrm{mmHg} \\
(\mathrm{N}= \\
7,315)\end{array}$ & $\begin{array}{l}10- \\
11 \mathrm{mmHg} \\
(\mathrm{N}= \\
42,878)\end{array}$ & $\begin{array}{l}12- \\
13 \mathrm{mmHg} \\
(\mathrm{N}= \\
59,851)\end{array}$ & $\begin{array}{l}14- \\
15 \mathrm{mmHg} \\
(\mathrm{N}= \\
29,422)\end{array}$ & $\begin{array}{l}\geq \\
16 \mathrm{mmHg} \\
(\mathrm{N}= \\
16,516)\end{array}$ & \\
\hline \multicolumn{7}{|l|}{$\begin{array}{l}\text { Baseline } \\
\text { characteristics }\end{array}$} \\
\hline Male & $47.6 \%$ & $47.6 \%$ & $44.9 \%$ & $44.1 \%$ & $41.4 \%$ & $45.2 \%$ \\
\hline Age, years & $58.3 \pm 9.2$ & $61.6 \pm 9.8$ & $64.2 \pm 9.8$ & $65.9 \pm 9.7$ & $67.2 \pm 9.7$ & $\begin{array}{l}63.9 \pm \\
10.0\end{array}$ \\
\hline Current smoker & $13.6 \%$ & $11.4 \%$ & $9.8 \%$ & $9.5 \%$ & $9.3 \%$ & $10.3 \%$ \\
\hline SBP, mmHg & $\begin{array}{l}114.3 \pm \\
9.3\end{array}$ & $\begin{array}{l}128.4 \pm \\
11.1\end{array}$ & $\begin{array}{l}138.4 \pm \\
13.8\end{array}$ & $\begin{array}{l}143.7 \pm \\
17.9\end{array}$ & $\begin{array}{l}149.6 \pm \\
25.2\end{array}$ & $\begin{array}{l}136.7 \pm \\
17.6\end{array}$ \\
\hline $\mathrm{DBP}, \mathrm{mmHg}$ & $69.2 \pm 8.1$ & $74.0 \pm 9.2$ & $75.9 \pm 9.8$ & $\begin{array}{l}76.4 \pm \\
10.8\end{array}$ & $\begin{array}{l}77.2 \pm \\
12.4\end{array}$ & $\begin{array}{l}75.3 \pm \\
10.2\end{array}$ \\
\hline $\mathrm{HbA} 1 \mathrm{c}, \%$ & $7.3 \pm 1.5$ & $7.4 \pm 1.4$ & $7.4 \pm 1.5$ & $7.4 \pm 1.5$ & $7.4 \pm 1.5$ & $7.4 \pm 1.4$ \\
\hline $\mathrm{BMI}, \mathrm{kg} / \mathrm{m}^{2}$ & $24.6 \pm 4.4$ & $25.4 \pm 4.4$ & $25.7 \pm 4.5$ & $25.7 \pm 5.5$ & $25.8 \pm 4.8$ & $\begin{array}{l}25.6 \pm \\
4.9\end{array}$ \\
\hline LDL-C, mmol/L & $3.1 \pm 0.9$ & $3.1 \pm 1.0$ & $3.2 \pm 1.0$ & $3.2 \pm 1.0$ & $3.2 \pm 1.0$ & $3.2 \pm 1.1$ \\
\hline $\begin{array}{l}\text { eGFR, } \\
\mathrm{ml} / \mathrm{min} / 1.73 \mathrm{~m}^{2}\end{array}$ & $\begin{array}{l}109.3 \pm \\
33.4\end{array}$ & $\begin{array}{l}104.0 \pm \\
41.6\end{array}$ & $\begin{array}{l}99.8 \pm \\
73.9\end{array}$ & $\begin{array}{l}96.3 \pm \\
47.4\end{array}$ & $\begin{array}{l}92.5 \pm \\
46.8\end{array}$ & $\begin{array}{l}100.0 \pm \\
62.1\end{array}$ \\
\hline Charlson Index & $2.4 \pm 1.1$ & $2.7 \pm 1.2$ & $3.0 \pm 1.2$ & $3.2 \pm 1.3$ & $3.4 \pm 1.3$ & $3.0 \pm 1.3$ \\
\hline $\begin{array}{l}\text { Use of oral anti- } \\
\text { diabetic drugs }\end{array}$ & $79.7 \%$ & $80.5 \%$ & $81.5 \%$ & $81.0 \%$ & $80.9 \%$ & $81.0 \%$ \\
\hline Use of insulin & $1.1 \%$ & $0.7 \%$ & $0.9 \%$ & $1.2 \%$ & $1.7 \%$ & $1.0 \%$ \\
\hline Use of ACEI/ARB & $11.1 \%$ & $20.4 \%$ & $31.9 \%$ & $39.2 \%$ & $44.6 \%$ & $30.5 \%$ \\
\hline Use of $\beta$-blocker & $16.8 \%$ & $23.5 \%$ & $29.2 \%$ & $34.9 \%$ & $44.6 \%$ & $29.8 \%$ \\
\hline
\end{tabular}

All parameters are expressed in either percentage or mean (sd). Other anti-hypertensive drugs included Acarbose, Glucagon-like peptide-1 agonist, Meglitinides. BMI = Body Mass Index; SBP = Systolic Blood Pressure; DBP = Diastolic Blood Pressure; HbA1c = Haemoglobin A1c; LDL-C = Lowdensity Lipoprotein-Cholesterol; eGFR = Estimated Glomerular Filtration Rate; ACEI = Angiotensin Converting Enzyme Inhibitor; $\mathrm{ARB}=$ Angiotensin Receptor Blocker; $\mathrm{CCB}$ = Calcium Channel Blocker;

$\mathrm{SD}=$ Standard deviation; 


\begin{tabular}{|c|c|c|c|c|c|c|}
\hline & \multicolumn{5}{|c|}{ SBP variability } & \multirow{2}{*}{$\begin{array}{l}\text { Overall } \\
(\mathrm{N}= \\
155,982)\end{array}$} \\
\hline & $\begin{array}{l}< \\
10 \mathrm{mmHg} \\
(\mathrm{N}= \\
7,315)\end{array}$ & $\begin{array}{l}10- \\
11 \mathrm{mmHg} \\
(\mathrm{N}= \\
42,878)\end{array}$ & $\begin{array}{l}12- \\
13 \mathrm{mmHg} \\
(\mathrm{N}= \\
59,851)\end{array}$ & $\begin{array}{l}14- \\
15 \mathrm{mmHg} \\
(\mathrm{N}= \\
29,422)\end{array}$ & $\begin{array}{l}\geq \\
16 \mathrm{mmHg} \\
(\mathrm{N}= \\
16,516)\end{array}$ & \\
\hline Use of CCB & $17.3 \%$ & $29.0 \%$ & $37.7 \%$ & $47.4 \%$ & $59.2 \%$ & $38.4 \%$ \\
\hline Use of Diuretic & $7.9 \%$ & $12.3 \%$ & $14.4 \%$ & $15.1 \%$ & $15.9 \%$ & $13.8 \%$ \\
\hline $\begin{array}{l}\text { Use of other anti- } \\
\text { hypertensive drugs }\end{array}$ & $5.1 \%$ & $7.6 \%$ & $10.8 \%$ & $13.5 \%$ & $17.1 \%$ & $10.8 \%$ \\
\hline $\begin{array}{l}\text { Use of lipid-lowering } \\
\text { agents }\end{array}$ & $7.9 \%$ & $9.8 \%$ & $9.8 \%$ & $10.0 \%$ & $10.6 \%$ & $9.8 \%$ \\
\hline \multicolumn{7}{|l|}{$\begin{array}{l}\text { Variables assessed } \\
\text { during } \\
\text { the assessment period }\end{array}$} \\
\hline $\begin{array}{l}\text { Number of SBP } \\
\text { measurements }\end{array}$ & $4.7 \pm 1.5$ & $4.6 \pm 0.6$ & $4.5 \pm 0.4$ & $4.5 \pm 0.6$ & $4.6 \pm 0.9$ & $4.5 \pm 1.3$ \\
\hline Usual SBP, mmHg & $\begin{array}{l}116.8 \pm \\
6.9\end{array}$ & $\begin{array}{l}129.6 \pm \\
3.0\end{array}$ & $\begin{array}{l}138.5 \pm \\
2.5\end{array}$ & $\begin{array}{l}143.3 \pm \\
3.8\end{array}$ & $\begin{array}{l}149.2 \pm \\
5.8\end{array}$ & $\begin{array}{l}137.1 \pm \\
10.7\end{array}$ \\
\hline SBP variability, mmHg & $9.0 \pm 0.4$ & $10.7 \pm 0.2$ & $12.5 \pm 0.2$ & $14.3 \pm 0.3$ & $17.4 \pm 1.2$ & $\begin{array}{l}12.7 \pm \\
2.3\end{array}$ \\
\hline \multicolumn{7}{|c|}{$\begin{array}{l}\text { All parameters are expressed in either percentage or mean (sd). Other anti-hypertensive drugs } \\
\text { included Acarbose, Glucagon-like peptide-1 agonist, Meglitinides. BMI = Body Mass Index; SBP = } \\
\text { Systolic Blood Pressure; DBP = Diastolic Blood Pressure; HbA1c = Haemoglobin A1c; LDL-C = Low- } \\
\text { density Lipoprotein-Cholesterol; } \mathrm{EGFR}=\text { Estimated Glomerular Filtration Rate; ACEI = Angiotensin } \\
\text { Converting Enzyme Inhibitor; ARB = Angiotensin Receptor Blocker; CCB = Calcium Channel Blocker; }\end{array}$} \\
\hline SD $=$ Standard deviatio & & & & & & \\
\hline
\end{tabular}

Table 2 demonstrates the cumulative incidence and incidence rate of CVD, all-cause mortality, and their composite events by SBP variability group. After a median follow-up of 9.7 years (16.4 million personyears), the overall number of any event is 49,816 incidents, incorporating 34,039 CVD and 29,211 mortalities. There was a trend for both cumulative incidence and incidence rates of all outcomes to increase with the SBP variability, from the lowest group (SBP variability $<10 \mathrm{mmHg}$ ) to the highest group (SBP variability $\geq 18 \mathrm{mmHg}$ ), including CVD from 12.8 to 42.7 , mortality from 10.3 to 34.2 , and all events from 20.6 to 60.9 per 1,000 person-years. Figure S3 presents the positive and log-linear association between SBP variability and all outcomes after adjustment for the patient's baseline characteristics. The results of the restricted cubic spline SBP variability association in the Cox models also suggested direct log-linear associations as indicated in Figure $\mathrm{S} 4$. The similar pattern by using other variability measurements such as coefficient of variation and variability independent of mean rather than SD were obtained. Age-specific associations are shown in Fig. 1 and Figure S2. As a whole, positive and log-linear 
associations are found between SBP and the risk of CVD, including CHD, stroke, heart failure, all-cause mortality, CVD mortality, non-CVD mortality and their composite events for all the age groups. 
Table 2

Number, incidence rate and hazard ratio of CVD and mortality, stratified by SBP variability

\section{SBP variability}

$\begin{array}{lllll}<10 \mathrm{mmHg} & 10- & 13- & 16- & \geq 18 \mathrm{mmHg} \\ (\mathrm{N}=7,315) & 12 \mathrm{mmHg} & 15 \mathrm{mmHg} & 18 \mathrm{mmHg} & (\mathrm{N}=16,516) \\ & (\mathrm{N}=42,878) & (\mathrm{N}=59,851) & (\mathrm{N}=29,422) & \end{array}$

CVD

\begin{tabular}{|c|c|c|c|c|c|}
\hline $\begin{array}{l}\text { Cumulative cases with } \\
\text { event }\end{array}$ & 819 & 7,306 & 13,031 & 7,731 & 5,152 \\
\hline $\begin{array}{l}\text { Incidence rate (95\% } \\
\mathrm{Cl}) \dagger\end{array}$ & $\begin{array}{l}12.8 \\
(12.0,13.8)\end{array}$ & $\begin{array}{l}20.1 \\
(19.6,20.6)\end{array}$ & $\begin{array}{l}26.6 \\
(26.1,27.0)\end{array}$ & $\begin{array}{l}33.7 \\
(32.9,34.4)\end{array}$ & $\begin{array}{l}42.7 \\
(41.5,43.9)\end{array}$ \\
\hline Hazard ratioł $(95 \% \mathrm{Cl})$ & $\begin{array}{l}1.00 \\
(0.92,1.08)\end{array}$ & $\begin{array}{l}1.18 \\
(1.15,1.21)\end{array}$ & $\begin{array}{l}1.27 \\
(1.25,1.29)\end{array}$ & $\begin{array}{l}1.41 \\
(1.37,1.44)\end{array}$ & $\begin{array}{l}1.58 \\
(1.52,1.64)\end{array}$ \\
\hline
\end{tabular}

Coronary heart

disease

\begin{tabular}{|c|c|c|c|c|c|}
\hline $\begin{array}{l}\text { Cumulative cases with } \\
\text { event }\end{array}$ & 417 & 3,543 & 6,093 & 3,513 & 2,331 \\
\hline $\begin{array}{l}\text { Incidence rate (95\% } \\
\mathrm{Cl}) \dagger\end{array}$ & $6.4(5.8,7.0)$ & $9.4(9.1,9.7)$ & $\begin{array}{l}11.8 \\
(11.5,12.1)\end{array}$ & $\begin{array}{l}14.3 \\
(13.8,14.7)\end{array}$ & $\begin{array}{l}17.7 \\
(17.0,18.5)\end{array}$ \\
\hline Hazard ratioł $(95 \% \mathrm{Cl})$ & $\begin{array}{l}1.00 \\
(0.90,1.12)\end{array}$ & $\begin{array}{l}1.15 \\
(1.11,1.20)\end{array}$ & $\begin{array}{l}1.19 \\
(1.17,1.22)\end{array}$ & $\begin{array}{l}1.27 \\
(1.23,1.33)\end{array}$ & $\begin{array}{l}1.42 \\
(1.35,1.50)\end{array}$ \\
\hline
\end{tabular}

Stroke

$\begin{array}{llllll}\text { Cumulative cases with } & 381 & 3,614 & 6,500 & 3,990 & 2,667\end{array}$ event

\begin{tabular}{|c|c|c|c|c|c|}
\hline $\begin{array}{l}\text { Incidence rate }(95 \% \\
\mathrm{Cl})+\end{array}$ & $5.8(5.3,6.4)$ & $9.6(9.2,9.9)$ & $\begin{array}{l}12.6 \\
(12.3,12.9)\end{array}$ & $\begin{array}{l}16.3 \\
(15.8,16.8)\end{array}$ & $\begin{array}{l}20.5 \\
(19.8,21.3)\end{array}$ \\
\hline Hazard ratio $\neq(95 \% \mathrm{Cl})$ & $\begin{array}{l}1.00 \\
(0.89,1.12)\end{array}$ & $\begin{array}{l}1.26 \\
(1.21,1.31)\end{array}$ & $\begin{array}{l}1.39 \\
(1.36,1.42)\end{array}$ & $\begin{array}{l}1.59 \\
(1.53,1.65)\end{array}$ & $\begin{array}{l}1.81 \\
(1.72,1.90)\end{array}$ \\
\hline \multicolumn{6}{|l|}{ Heart failure } \\
\hline $\begin{array}{l}\text { Cumulative cases with } \\
\text { event }\end{array}$ & 126 & 1,693 & 3,726 & 2,406 & 1,821 \\
\hline $\begin{array}{l}\text { Incidence rate (95\% } \\
\mathrm{Cl}) \dagger\end{array}$ & $1.9(1.6,2.3)$ & $4.4(4.2,4.6)$ & $7.0(6.8,7.3)$ & $9.5(9.2,9.9)$ & $\begin{array}{l}13.6 \\
(12.9,14.2)\end{array}$ \\
\hline Hazard ratio $\neq(95 \% \mathrm{Cl})$ & $\begin{array}{l}1.00 \\
(0.82,1.23)\end{array}$ & $\begin{array}{l}1.38 \\
(1.30,1.46)\end{array}$ & $\begin{array}{l}1.48 \\
(1.44,1.53)\end{array}$ & $\begin{array}{l}1.56 \\
(1.49,1.64)\end{array}$ & $\begin{array}{l}1.76 \\
(1.65,1.87)\end{array}$ \\
\hline
\end{tabular}

$\mathrm{SD}=$ Standard deviation; $\mathrm{SBP}=$ Systolic blood pressure $; \mathrm{CVD}=$ Cardiovasular disease $; \mathrm{CKD}=$ Chronic kidney disease; $\mathrm{Cl}=$ Confidence interval 


\section{SBP variability}

\begin{tabular}{|c|c|c|c|c|c|}
\hline \multicolumn{6}{|l|}{ All cause mortality } \\
\hline $\begin{array}{l}\text { Cumulative cases with } \\
\text { event }\end{array}$ & 689 & 5,976 & 11,070 & 6,707 & 4,769 \\
\hline $\begin{array}{l}\text { Incidence rate }(95 \% \\
\mathrm{Cl}) \dagger\end{array}$ & $\begin{array}{l}10.3 \\
(9.6,11.1)\end{array}$ & $\begin{array}{l}15.3 \\
(14.9,15.6)\end{array}$ & $\begin{array}{l}20.5 \\
(20.1,20.9)\end{array}$ & $\begin{array}{l}25.9 \\
(25.3,26.5)\end{array}$ & $\begin{array}{l}34.2 \\
(33.3,35.2)\end{array}$ \\
\hline Hazard ratio $\neq(95 \% \mathrm{Cl})$ & $\begin{array}{l}1.00 \\
(0.91,1.09)\end{array}$ & $\begin{array}{l}1.06 \\
(1.03,1.10)\end{array}$ & $\begin{array}{l}1.14 \\
(1.12,1.16)\end{array}$ & $\begin{array}{l}1.25 \\
(1.21,1.29)\end{array}$ & $\begin{array}{l}1.46 \\
(1.40,1.52)\end{array}$ \\
\hline
\end{tabular}

Cardiovasular

mortality

\begin{tabular}{llllll}
$\begin{array}{l}\text { Cumulative cases with } \\
\text { event }\end{array}$ & 209 & 2,501 & 5,186 & 3,441 & 2,670 \\
\hline $\begin{array}{l}\text { Incidence rate }(95 \% \\
\text { Cl)† }\end{array}$ & $3.1(2.7,3.6)$ & $6.4(6.1,6.6)$ & $9.6(9.3,9.9)$ & $\begin{array}{l}13.3 \\
(12.9,13.8)\end{array}$ & $\begin{array}{l}19.2 \\
(18.5,19.9)\end{array}$ \\
\hline Hazard ratioł $(95 \% \mathrm{Cl})$ & $\begin{array}{l}1.00 \\
(0.85,1.18)\end{array}$ & $\begin{array}{l}1.26 \\
(1.20,1.33)\end{array}$ & $\begin{array}{l}1.38 \\
(1.34,1.42)\end{array}$ & $\begin{array}{l}1.59 \\
(1.52,1.66)\end{array}$ & $\begin{array}{l}1.90 \\
(1.79,2.01)\end{array}$
\end{tabular}

Non-cardiovasular

mortality

\begin{tabular}{llllll}
$\begin{array}{l}\text { Cumulative cases with } \\
\text { event }\end{array}$ & 480 & 3,475 & 5,884 & 3,266 & 2,099 \\
\hline $\begin{array}{l}\text { Incidence rate }(95 \% \\
\text { Cl)† }\end{array}$ & $7.2(6.6,7.8)$ & $8.9(8.6,9.2)$ & $\begin{array}{l}10.9 \\
(10.6,11.2)\end{array}$ & $\begin{array}{l}12.6 \\
(12.2,13.1)\end{array}$ & $\begin{array}{l}15.1 \\
(14.5,15.7)\end{array}$ \\
\hline Hazard ratioł $(95 \% \mathrm{Cl})$ & $\begin{array}{l}1.00 \\
(0.90,1.11)\end{array}$ & $\begin{array}{l}1.02 \\
(0.97,1.06)\end{array}$ & $\begin{array}{l}1.08 \\
(1.06,1.11)\end{array}$ & $\begin{array}{l}1.13 \\
(1.09,1.18)\end{array}$ & $\begin{array}{l}1.26 \\
(1.19,1.34)\end{array}$
\end{tabular}

All composite events

$\begin{array}{llllll}\begin{array}{l}\text { Cumulative cases with } \\ \text { event }\end{array} & 1,311 & 10,893 & 19,129 & 11,138 & 7,345\end{array}$

\begin{tabular}{|c|c|c|c|c|c|}
\hline $\begin{array}{l}\text { Incidence rate }(95 \% \\
\mathrm{Cl}) \dagger\end{array}$ & $\begin{array}{l}20.6 \\
(19.5,21.7)\end{array}$ & $\begin{array}{l}30.0 \\
(29.4,30.5)\end{array}$ & $\begin{array}{l}39.0 \\
(38.4,39.5)\end{array}$ & $\begin{array}{l}48.5 \\
(47.6,49.4)\end{array}$ & $\begin{array}{l}60.9 \\
(59.5,62.3)\end{array}$ \\
\hline $\mathrm{Ha}$ & $\begin{array}{l}1.00 \\
(0.94,1.07)\end{array}$ & $\begin{array}{l}1.13 \\
(1 \cdot 10,1 \cdot 15)\end{array}$ & $\begin{array}{l}1.22 \\
(1.20,1.23)\end{array}$ & $\begin{array}{l}1.33 \\
(1.30,1.36)\end{array}$ & $\begin{array}{l}1.50 \\
(1.46,1.55)\end{array}$ \\
\hline
\end{tabular}

$\mathrm{SD}=$ Standard deviation; $\mathrm{SBP}=$ Systolic blood pressure; $\mathrm{CVD}=$ Cardiovasular disease $; \mathrm{CKD}=$ Chronic kidney disease; $\mathrm{Cl}=$ Confidence interval

Figure 2 demonstrates the relationship between SBP variability as a continuous variable and the risk of all outcome events. Each $5 \mathrm{mmHg}$ increase in SBP variability was associated with $27 \%$ (HR: 1.27 [95\% Cl 1.23-1.32]), 30\% (HR: 1.30 [95\% Cl 1.25-1.35]), and 26\% (HR: 1.26 [95\% Cl 1.23-1.30]) higher risk of CVD, all-cause mortality, and their composite, respectively. Moreover, the HR for stroke (HR: 1.33 [95\% Cl 
1.27-1.40]) is higher than that for CHD (HR: 1.21 [95\% Cl 1.15-1.27]) and heart failure (HR: 1.21 [95\% Cl 1.14-1.28]), indicating that the association with CVD may be driven predominantly by stroke. Similarly, the HR for non-CVD related mortality (HR: 1.18 [95\% Cl 1.12-1.25]) was lower than that for CVD-related mortality (HR: 1.40 [95\% Cl 1.33-1.48]), implying that mortality may be mainly attributable to CVD-related mortality. Four sensitivity analyses including (1) the use of Rosner's regression method in Figure S5, (2) complete case analysis in Figure S6, (3) excluding patients with less than 1-year follow-up period in Figure S7, (4) including patients with $\geq 2$ and $\geq 7$ rather than $\geq 4$ SBP measurements in Figure S8-S9, (5) including the usage of aspirin on or before baseline in Figure $\mathrm{S} 10$, demonstrated similar results.

The forest plots in Fig. 3 and Figure S11-S12 show that SBP variability is associated with a significant increase in the risk of each outcome event across all selected subgroups. In general, the HRs for the composite of CVD and all-cause mortality demonstrate that age and usual SBP is inversely related with the SBP variability HR. The HRs are higher among patients with low comorbidity (Charlson's index $<4$ ), and patient without anti-hypertensive drug prescribed. Comparable effects are observed when stratified by male, smoking status, BMI, baseline SBP, HbA1c, eGFR, and LDL-C, and use of diverse antihypertensive drugs. The age at risk subgroups have the most pronounced difference in SBP variability HRs for the risk of CVD and all-cause mortality, with a $66 \%$ higher risk per $5 \mathrm{mmHg}$ increase in the 4554 year group [all composite outcomes HR: $1.66(1.49,1.85)$ ], compared to a $19 \%$ higher risk in the $70-$ 79 year group [all composite outcomes HR: $1.19(1.15,1.23)]$, which indicates that the risk in the 4554 year age group is over three times higher than the 70-79 year group. Similar trend for the usual SBP were obtained that patients with usual SBP $<130 \mathrm{mmHg}$ had double the risk compared to those with usual SBP $\geq 150 \mathrm{mmHg}$ [HR: $1.39(1.29,1.49)$ vs $1.17(1.11,1.23)]$. Different effect of SBP variability were observed in terms of the comorbidity [HR: $1.36(1.32,1.41)$ in Charlson's index < 4 vs $1.23(1.19$, 1.27) in Charlson's index $\geq 4$ ] and number of type of anti-hypertensive drug prescribed [HR: 1.49 (1.39, $1.60)$ in none vs $1.27(1.21,1.33)$ in $\geq 3$ types].

\section{Discussion}

This large cohort study is the first to estimate SBP variability using mixed effects models to reduce regression dilution, and to determine the positive and log-linear age-specific association between SBP variability and risk of CVD, CHD, stroke, heart failure, all-cause mortality, CVD mortality, and non-CVD mortality among Chinese diabetic patients. The effect of SBP variability on the risk of various outcome events remained significant regardless of patient characteristics after adjusting for usual and baseline SBP, suggesting that SBP variability may provide additional valuable information as a potential predictor for the incidence of CVD events and mortality in the diabetic population, irrespective of the absolute SBP readings. There was no threshold for SBP variability, indicating that the lower the SBP variability, the better the outcome. These findings recommend that clinicians should be cautious about the effect of BP fluctuation on the incidence of CVD and all-cause mortality, as well as the focus on an optimal BP target. Moreover, the impact of SBP variability is strengthened in patients at younger age, lower usual SBP, Charlson's index and number of types of anti-hypertensive drug prescribed. This is suggestive that additional attention may be needed for these specific patients; groups with higher SBP variability. 
There is currently no literature investigating the age-specific effects of SBP variability on CVD and mortality amongst diabetic patients. In general, our results demonstrated a direct log-linear or linear association between SBP variability and outcome events, which confirmed the results of previous studies. For instance, a post-trial follow-up study of the landmark trial, named Action in Diabetes and Vascular Disease: Preterax and Diamicron Modified Release Controlled Evaluation (ADVANCE) trial, on 9,113 diabetic patients with a medium follow-up of 7.6 years also illustrated the positive log-linear associations that each $5 \mathrm{mmHg}$ increment in SD of SBP was associated with a $13 \%, 8 \%$ and $11 \%$ increase in the risk of all-cause mortality, CVD, and myocardial infarction, respectively (15). No significant association for stroke and CVD mortality was obtained (15). Nevertheless, they acknowledged that the small sample size and number of events (668 stroke events and 614 CVD mortality events) may affect the generalizability of the results. A few studies have shown a lack of any significant associations between SBP variability and outcome events $(11,35)$. In a cohort study on 2,161 diabetic patients in Taiwan, over a 5.5 year follow-up, SBP variability was significant associated with all-cause mortality but not with CVD mortality. This was likely to be explained by the small sample size and small number of events as only 25 CVD mortality events among 2,161 patients were observed (35). Furthermore, the measurement of SBP variability was determined by SBP readings after baseline, which may have resulted in informative censoring or immortal time bias $(12,13)$. Compared to these studies, we have incorporated a significantly larger number of patients without CVD, longer follow-up periods and much large number of incident outcome events. In this current study, SBP variability was based on measurements performed before baseline, hence the results should be more reliable and better powered to demonstrate the etiological associations between SBP variability and adverse outcomes. It is also worthy to mention that the results in all previous studies did not correct for regression dilution bias, and thus their findings may underestimate the effect of SBP variability. Hence, it is expected that the strength of the association of SBP variability on the risk of event outcomes in this study will be greater.

In this current study the analyses of individual CVD events, identified a stronger impact of SBP variability on stroke compared to CHD and heart failure. Indeed, the previous meta-analysis, which included mostly studies involving patients with severe diseases such as CVD or from the general population, also showed a similar pattern that SBP variability, as with usual blood pressure (30), contributes more to stroke than cardiac events (12). Current findings extended these findings that the effect of SBP variability on CVD event may be driven primarily by stroke in the diabetic population.

The pathophysiological mechanisms underlying the association between SBP variability and risks of CVD and mortality has not been fully clarified. On one hand, augmented BP variability is associated with endothelial dysfunction and chronic inflammation (36-39) which may speed up atherosclerosis, eventually leading to the development of CVD and increased mortality risk $(36,37)$. The increased variability in BP could also lead to arterial stiffness, which makes one prone to encounter adverse vascular events (40-42). Other possible mechanisms include coronary artery calcification, left ventricular diastolic dysfunction, and glomerulosclerosis, which were all in the direction of negative clinical outcomes $(40,41,43,44)$. In addition, persistent fluctuations in BP could be a reflection of abnormal autonomic regulation from end-organ damage as has been reported in animal models.(37) 
A recent post-hoc analysis of the Valsartan Antihypertensive Long-term Use Evaluation trial (VALUE) on 13,803 hypertensive patients over a mean follow-up period of 4.2 years reported that SBP variability was associated with a significantly higher risk of multiple diseases in patients with age < 68 years than in those with age $\geq 68$ years (45). On top of this observation, our findings from subgroup analyses showed a trend that not only within the age range from 45 to 85 years that younger people was more susceptible to the adverse influence of SBP variability but also the impact was stronger on lower usual SBP, Charlson's index and number of types of anti-hypertensive drug prescribed. The reasons may be that these patients were more likely to have higher SBP variability, as shown in Table 1 and literature (46). Therefore, these groups may tolerate more continual fluctuation than others. As a consequence, these patients may be more vulnerable to SBP variability. Meanwhile, patients with these conditions (e.g. younger and low comorbidity) were more likely with fewer vascular risk factors, and thus may be more sensitive to blood pressure variability. On the contrary, other risk factors in patients with higher age and morbidity may overshadow the detrimental effect of SBP variability. A few studies concluded that poor anti-hypertensive medication adherence and usage of different types of anti-hypertensive drugs may cause patients to be more prone to higher SBP variability and CVD risk (47-50). Although drug adherence information was unavailable in this current study, many previous studies have found no significant difference in SBP variability amongst different levels of drug adherence or types of anti-hypertensive medications used (51-53). Therefore, these factors do not explain the links between SBP variability and the incidence of CVD and all-cause mortality $(46,52)$. Further studies are required to clarify the mechanism of this relationship.

\section{Strengths and Limitations of this study}

Our study has several strengths. Firstly, we included a Type 2 Diabetes cohort with a near ten-year followup period, which was the largest and longest study and well powered to demonstrate the associations between SBP variability and outcome events in different subgroups. Secondly, we used appropriate statistical analysis methods to correct for regression dilution bias and conducted sensitivity analyses, which allowed us to make a comprehensive evaluation of the relationship between SBP and diverse clinical outcomes. Multiple imputations were used to impute missing data in order to reduce selection bias. Thirdly, relevant baseline covariates, such as patients' laboratory results, disease attributes, and treatment modalities, were considered to generate reliable results with the aid of HA's computerised administrative database.

There were also limitations to our study. Firstly, the study design of a retrospective cohort study can only yield a conclusion about association but not causation. However, a low probability of reverse causation was observed as patients with CVD at baseline were excluded in this study, the results were very similar in the sensitivity analysis when we only included patients with a follow-up period of above one year. Secondly, potential confounding factors related to lifestyles, such as physical activity level and dietary intake, were not assessed in this study. Instead, we have examined the individuals' anthropometric and clinical parameters, including BMI, $\mathrm{HbA} 1 \mathrm{c}$, and lipid profile, which could alternatively reflect the severity of 
their diseases and their lifestyle habits. Lastly, the association between SBP variability and increased CVD risk was well demonstrated in this study. However, this could possibly be due to the individual differences amongst our study subjects, compared to the general population and the Type 2 diabetic populations from other Chinese regions. Temporal variations and alterations in non-assessed risk factors or interventions might also induce heterogeneity in the association. Therefore, our findings might not be applicable to other settings.

\section{Conclusion}

In this population-based cohort study of Chinese primary care patients with Type 2 diabetes, SBP variability was associated with increased CVD risk and all-cause mortality regardless of usual and baseline SBP. We demonstrated positive log-linear age-specific associations between SBP variability and the risk of CVD, CHD, stroke, heart failure, all-cause mortality, CVD mortality, and non-CVD mortality. The clinical implication of these findings is that the SBP variability should be monitored and evaluated in routine practice, as this appears to be a risk factor for developing CVD and all-cause mortality. The impact of SBP variability was more significant in patients with younger age, lower usual SBP and comorbidity, and high number of types of anti-hypertensive prescription drug users. We suggest that additional monitoring may be needed for these patients if they have higher SBP variability. Future studies are needed to standardise the definition of the variability of BP and to determine a therapeutic target for SBP variability to provide better outcomes for patients with Type 2 diabetes.

\section{List Of Abbreviations}

Diabetes Mellitus (DM)

Cardiovascular disease (CVD)

Systolic blood pressure (SBP)

Preterax and Diamicron Modified Release Controlled Evaluation (ADVANCE)

Randomized controlled trial (RCT)

Hospital Authority (HA)

International Classification of Primary Care-2 (ICPC-2)

Clinical management system (CMS)

Coronary heart disease (CHD)

International Classification of Diseases, Ninth Edition, Clinical Modification (ICD-9-CM)

Confidence interval (Cl)

Page $15 / 23$ 
Body mass index (BMI)

Haemoglobin A1c (HbA1c)

Low-density lipoprotein-cholesterol (LDL-C)

Estimated glomerular filtration rate (eGFR)

angiotensin receptor blocker (ACEI/ARB)

Calcium channel blocker (CCB)

Valsartan Antihypertensive Long-term Use Evaluation trial (VALUE)

\section{Declarations}

\section{Ethics approval and consent to participate}

All data in this study are anonymous and extracted from the clinical database in Hong Kong Hospital Authority, and thus the informed consent from all study subjects is not required. Ethics approval was granted by the Institutional Review Boards (IRB) of the Hong Kong Hospital Authority.

\section{Consent for publication}

Not applicable.

\section{Availability of data and materials}

The data for this study is hosted by the Hong Kong Hospital Authority. Subject to local law and regulation regarding the use and distribution personal data, the database used in the present study cannot be deposited in public data repositories. Data can be applied through the Data Sharing Portal of the Hong Kong Hospital Authority (https://www3.ha.org.hk/data/DCL/Index/).

\section{Competing interests}

I.C.K.W. received funding from Pfizer, Bayer and Novartis to evaluate real world evidence on pharmacological treatments of cardiovascular diseases but not related to current study. E.W.Y.C. received research grants from Bayer, Bristol-Myers Squibb, Janssen, a Division of Johnson and Johnson, Pfizer, and Takeda to evaluate real world evidence on pharmacological treatments of cardiovascular diseases but not related to current study. Other authors declare that they have no competing interests.

\section{Funding}

This work was supported by the Health Services Research Fund, Food and Health Bureau, HKSAR (Ref. no 14151181). No funding organization had any role in the design and conduct of the study; collection, 
management, analysis, and interpretation of the data; and preparation of the manuscript.

\section{Author Contributions}

E.Y.F.W., and C.L.K.L. contributed to the study design and acquisition of data, researched the data, contributed to the statistical analysis and interpretation of the results, and wrote the manuscript. All authors contributed to the interpretation of the results, reviewed and edited the manuscript. E.Y.F.W. is the guarantor of this work and, as such, had full access to all the data in the study and takes responsibility for the integrity of the data and the accuracy of the data analysis.

\section{Authors' contributions}

EW, EY, WC, JB, IW, EC, CC, SC and CL contributed to the concept and rationale for this study. EW conducted statistical analysis. All authors were involved in data interpretation. EW, EY, WC, JB, IW, EC, CC, SC and CL contributed to discussion. EW, EY, WC, JB, IW, EC, CC, SC and CL drafted the manuscript. All authors reviewed and edited the manuscript and approved the final submitted version. EW, EY, WC, JB, IW, $\mathrm{EC}, \mathrm{CC}, \mathrm{SC}$ and $\mathrm{CL}$ are the guarantors of this work and, as such, can access all the data in this study and in charge of data integrity and the accuracy of the data analysis.

\section{Acknowledgements}

The authors wish to acknowledge the contributions of the Risk Assessment Management Program (RAMP) for program team at the Hospital Authority head office, and the Chiefs of Service and RAMP program coordinators in each cluster, and the Statistics and Workforce Planning Department at the Hong Kong Hospital Authority.

\section{References}

1. International Diabetes Federation. IDF DIABETES ATLAS Eighth edition 2017. Brussels, Belgium; 2017.

2. World Health Organization. Complications of diabetes 2019 [Available from: https://www.who.int/diabetes/action_online/basics/en/index3.html.

3. International Diabetes Federation. Diabetes and cardiovascular disease. Brussels, Belgium; 2016.

4. Bundy JD, Li C, Stuchlik P, Bu X, Kelly TN, Mills KT, et al. Systolic Blood Pressure Reduction and Risk of Cardiovascular Disease and Mortality: A Systematic Review and Network Meta-analysis. JAMA Cardiol. 2017;2(7):775-81.

5. Brunstrom M, Carlberg B. Association of Blood Pressure Lowering With Mortality and Cardiovascular Disease Across Blood Pressure Levels: A Systematic Review and Meta-analysis. JAMA Intern Med. 2018;178(1):28-36.

6. American Diabetes A. 8. Cardiovascular Disease and Risk Management. Diabetes Care. 2016;39 Suppl 1:S60-71. 
7. Emdin CA, Rahimi K, Neal B, Callender T, Perkovic V, Patel A. Blood pressure lowering in type 2 diabetes: a systematic review and meta-analysis. JAMA. 2015;313(6):603-15.

8. Association AD. 2. Classification and diagnosis of diabetes: standards of medical care in diabetes2019. Diabetes Care. 2019;42(Supplement 1):S13-S28.

9. Group IDFW. IDF clinical practice recommendations for managing type 2 diabetes in primary care. International Diabetes Federation Brussels; 2017.

10. Chiriacò M, Pateras K, Virdis A, Charakida M, Kyriakopoulou D, Nannipieri M, et al. Association between blood pressure variability, cardiovascular disease and mortality in type 2 diabetes: A systematic review and meta-analysis. Diabetes, Obesity and Metabolism.0(0).

11. Bell KJL, Azizi L, Nilsson PM, Hayen A, Irwig L, Ostgren CJ, et al. Prognostic impact of systolic blood pressure variability in people with diabetes. PLoS One. 2018;13(4):e0194084.

12. Stevens SL, Wood S, Koshiaris C, Law K, Glasziou P, Stevens RJ, et al. Blood pressure variability and cardiovascular disease: systematic review and meta-analysis. BMJ. 2016;354:i4098.

13. Lévesque LE, Hanley JA, Kezouh A, Suissa S. Problem of immortal time bias in cohort studies: example using statins for preventing progression of diabetes. Bmj. 2010;340:b5087.

14. Wan EYF, Fung CSC, Yu EYT, Fong DYT, Chen JY, Lam CLK. Association of Visit-to-Visit Variability of Systolic Blood Pressure With Cardiovascular Disease and Mortality in Primary Care Chinese Patients With Type 2 Diabetes-A Retrospective Population-Based Cohort Study. Diabetes Care. 2017;40(2):270-9.

15. Ohkuma T, Woodward M, Jun M, Muntner P, Hata J, Colagiuri S, et al. Prognostic Value of Variability in Systolic Blood Pressure Related to Vascular Events and Premature Death in Type 2 Diabetes Mellitus: The ADVANCE-ON Study. Hypertension. 2017;70(2):461-8.

16. Barrett JK, Huille R, Parker R, Yano Y, Griswold M. Estimating the association between blood pressure variability and cardiovascular disease: An application using the ARIC Study. Statistics in medicine. 2018.

17. Rapsomaniki E, Timmis A, George J, Pujades-Rodriguez M, Shah AD, Denaxas S, et al. Blood pressure and incidence of twelve cardiovascular diseases: lifetime risks, healthy life-years lost, and agespecific associations in 1·25 million people. The Lancet. 2014;383(9932):1899-911.

18. Rothwell PM. Limitations of the usual blood-pressure hypothesis and importance of variability, instability, and episodic hypertension. Lancet. 2010;375(9718):938-48.

19. Lau IT. A Clinical Practice Guideline to Guide a System Approach to Diabetes Care in Hong Kong. Diabetes \& Metabolism Journal. 2017;41(2):81-8.

20. Chan EW, Lau WC, Leung WK, Mok MT, He Y, Tong TS, et al. Prevention of dabigatran-related gastrointestinal bleeding with gastroprotective agents: a population-based study. Gastroenterology. 2015;149(3):586-95. e3.

21. Wong AY, Root A, Douglas IJ, Chui CS, Chan EW, Ghebremichael-Weldeselassie Y, et al. Cardiovascular outcomes associated with use of clarithromycin: population based study. bmj. 2016;352:h6926. 
22. Plummer M. JAGS Version 4.3. 0 user manual. 2017.

23. Su Y-S, Yajima M. R2jags: Using R to run 'JAGS'. R package version 05-7. 2015;34.

24. Charlson M, Szatrowski TP, Peterson J, Gold J. Validation of a combined comorbidity index. Journal of clinical epidemiology. 1994;47(11):1245-51.

25. Charlson ME, Pompei P, Ales KL, MacKenzie CR. A new method of classifying prognostic comorbidity in longitudinal studies: development and validation. Journal of chronic diseases. 1987;40(5):373-83.

26. Ma Y-C, Zuo L, Chen J-H, Luo Q, Yu X-Q, Li Y, et al. Modified glomerular filtration rate estimating equation for Chinese patients with chronic kidney disease. Journal of the American Society of Nephrology. 2006;17(10):2937-44.

27. Rubin DB. Multiple imputation for nonresponse in surveys: John Wiley \& Sons; 2004.

28. San H, Lewington S, editors. Lexis expansion-age-at-risk adjustment for survival analysis. Brussels: Statistics and Pharmacokinetics Pharmaceutical Users Software Exchange conference; 2013.

29. Plummer M. Improved estimates of floating absolute risk. Statistics in medicine. 2004;23(1):93-104.

30. Lacey B, Lewington S, Clarke R, Kong XL, Chen Y, Guo Y, et al. Age-specific association between blood pressure and vascular and non-vascular chronic diseases in 0.5 million adults in China: a prospective cohort study. The Lancet Global Health. 2018;6(6):e641-e9.

31. Collaboration PS. Age-specific relevance of usual blood pressure to vascular mortality: a metaanalysis of individual data for one million adults in 61 prospective studies. The Lancet. 2002;360(9349):1903-13.

32. Collaboration APCS. Blood pressure and cardiovascular disease in the Asia Pacific region. Journal of hypertension. 2003;21(4):707-16.

33. Durrleman S, Simon R. Flexible regression models with cubic splines. Statistics in medicine. 1989;8(5):551-61.

34. Rosner B, Willett W, Spiegelman D. Correction of logistic regression relative risk estimates and confidence intervals for systematic within-person measurement error. Statistics in medicine. 1989;8(9):1051-69.

35. Hsieh YT, Tu ST, Cho TJ, Chang SJ, Chen JF, Hsieh MC. Visit-to-visit variability in blood pressure strongly predicts all-cause mortality in patients with type 2 diabetes: a 5.5-year prospective analysis. Eur J Clin Invest. 2012;42(3):245-53.

36. Faramawi MF, Delongchamp R, Said Q, Jadhav S, Abouelenien S. Metabolic syndrome is associated with visit-to-visit systolic blood pressure variability in the US adults. Hypertension Research. 2014;37(9):875-9.

37. Su D-F. Treatment of hypertension based on measurement of blood pressure variability: Iessons from animal studies. Current opinion in cardiology. 2006;21(5):486-91.

38. Kim K-I, Lee J-H, Chang H-J, Cho Y-S, Youn T-J, Chung W-Y, et al. Association between blood pressure variability and inflammatory marker in hypertensive patients. Circulation Journal. 2008;72(2):293-8. 
39. Tatasciore A, Zimarino M, Renda G, Zurro M, Soccio M, Prontera C, et al. Awake blood pressure variability, inflammatory markers and target organ damage in newly diagnosed hypertension. Hypertension Research. 2008;31(12):2137.

40. Song H, Wei F, Liu Z, Zhao Y, Ye L, Lu F, et al. Visit-to-visit variability in systolic blood pressure: correlated with the changes of arterial stiffness and myocardial perfusion in on-treated hypertensive patients. Clinical and Experimental Hypertension. 2015;37(1):63-9.

41. Nagai M, Hoshide S, Ishikawa J, Shimada K, Kario K. Visit-to-visit blood pressure variations: new independent determinants for carotid artery measures in the elderly at high risk of cardiovascular disease. Journal of the American Society of Hypertension. 2011;5(3):184-92.

42. Okada H, Fukui M, Tanaka M, Inada S, Mineoka Y, Nakanishi N, et al. Visit-to-visit variability in systolic blood pressure is correlated with diabetic nephropathy and atherosclerosis in patients with type 2 diabetes. Atherosclerosis. 2012;220(1):155-9.

43. Shimbo D, Shea S, McClelland RL, Viera AJ, Mann D, Newman J, et al. Associations of aortic distensibility and arterial elasticity with long-term visit-to-visit blood pressure variability: the MultiEthnic Study of Atherosclerosis (MESA). American journal of hypertension. 2013:hpt040.

44. Parati G, Liu X, Ochoa J. Clinical relevance of visit-to-visit blood pressure variability: impact on renal outcomes. Journal of Human Hypertension. 2014;28(7):403.

45. Mehlum MH, Liestøl K, Kjeldsen SE, Julius S, Hua TA, Rothwell PM, et al. Blood pressure variability and risk of cardiovascular events and death in patients with hypertension and different baseline risks. European heart journal. 2018;39(24):2243-51.

46. Hata J, Arima H, Rothwell PM, Woodward M, Zoungas S, Anderson C, et al. Effects of visit-to-visit variability in systolic blood pressure on macrovascular and microvascular complications in patient with type 2 diabetes: the ADVANCE trial. Circulation. 2013:CIRCULATIONAHA. 113.002717.

47. Krakoff LR. Fluctuation: does blood pressure variability matter? Circulation. 2012:CIRCULATIONAHA. 112.124750 .

48. Hong K, Muntner P, Kronish I, Shilane D, Chang T. Medication adherence and visit-to-visit variability of systolic blood pressure in African Americans with chronic kidney disease in the AASK trial. Journal of human hypertension. 2015.

49. Wijsman LW, de Craen AJ, Muller M, Sabayan B, Stott D, Ford I, et al. Blood pressure lowering medication, visit-to-visit blood pressure variability, and cognitive function in old age. American journal of hypertension. 2015:hpv101.

50. Hong K, Muntner P, Kronish I, Shilane D, Chang TI. Medication adherence and visit-to-visit variability of systolic blood pressure in African Americans with chronic kidney disease in the AASK trial. Journal of human hypertension. 2016;30(1):73.

51. Kronish IM, Lynch Al, Oparil S, Whittle J, Davis BR, Simpson LM, et al. The association between antihypertensive medication nonadherence and visit-to-visit variability of blood pressure: findings from the Antihypertensive and Lipid-Lowering Treatment to Prevent Heart Attack Trial. Hypertension. 2016;68(1):39-45. 
52. Muntner P, Whittle J, Lynch Al, Colantonio LD, Simpson LM, Einhorn PT, et al. Visit-to-visit variability of blood pressure and coronary heart disease, stroke, heart failure, and mortality: a Cohort Study. Annals of internal medicine. 2015;163(5):329-38.

53. Shiga Y, Miura S-i, Adachi S, Suematsu Y, Sugihara M, Iwata A, et al. Visit-to-Visit Variability and Seasonal Variation in Blood Pressure With Single-Pill Fixed-Dose Combinations of Angiotensin II Receptor Blocker/Calcium Channel Blocker and Angiotensin II Receptor Blocker/Diuretic in Hypertensive Patients. Journal of clinical medicine research. 2015;7(10):802.

\section{Figures}
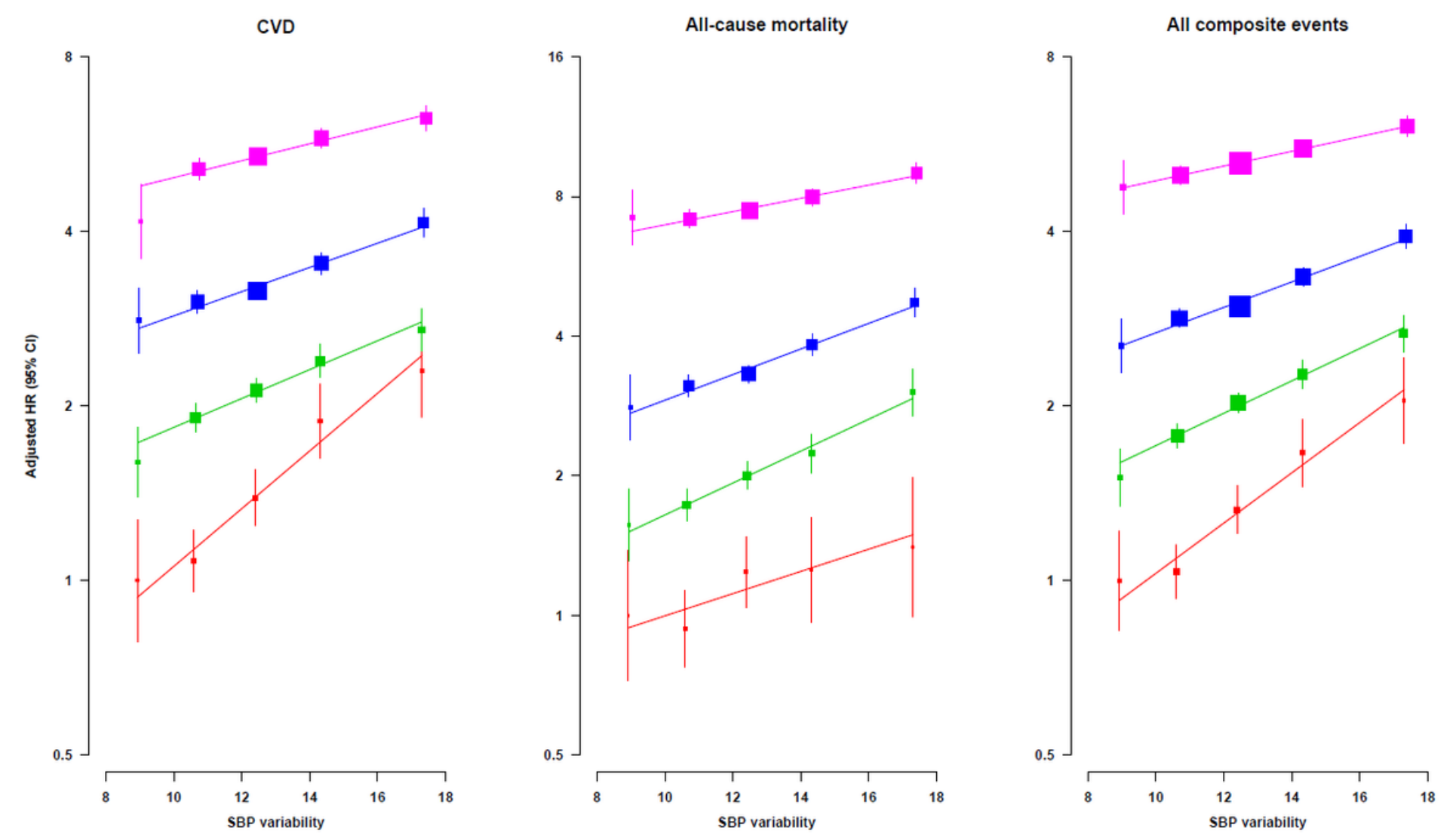

Figure 1

Age-specific adjusted hazard ratios for the risk of CVD, all-cause mortality and their composite with with increasing SBP variability by multivariable Cox regressions. HR was adjusted by age at risk, gender, smoking status, body mass index, SBP, diastolic blood pressure, haemoglobin A1c, low-density lipoprotein-cholesterol, estimated glomerular filtration rate, the usages of oral anti-diabetic drugs, insulin, angiotensin converting enzyme inhibitor/angiotensin receptor blocker, $\beta$-blocker, calcium channel blocker, diuretic, other anti-hypertensive drugs, lipid-lowering agent, Charlson's index and usual SBP. Red, green, blue and pink line were 45-54, 55-64, 65-74 and 75-84 age at risk group. The area of each square was inversely proportional to the variance of the category-specific log risk. Cls are displayed as floating 
absolute risks. $\mathrm{SBP}=$ Systolic blood pressure; $\mathrm{CVD}=$ Cardiovascular disease; $\mathrm{HR}=$ Hazard ratio; $\mathrm{Cl}=$ Confidence interval.

\section{Event}

\section{Outcome}

CVD

Coronary heart disease

Stroke

Heart failure

All cause mortality

Cardiovasular mortality

Non-cardiovasular mortality

All composite events
34,039

15,897

17,152

9,772

29,211

14,007

15,204

49,816

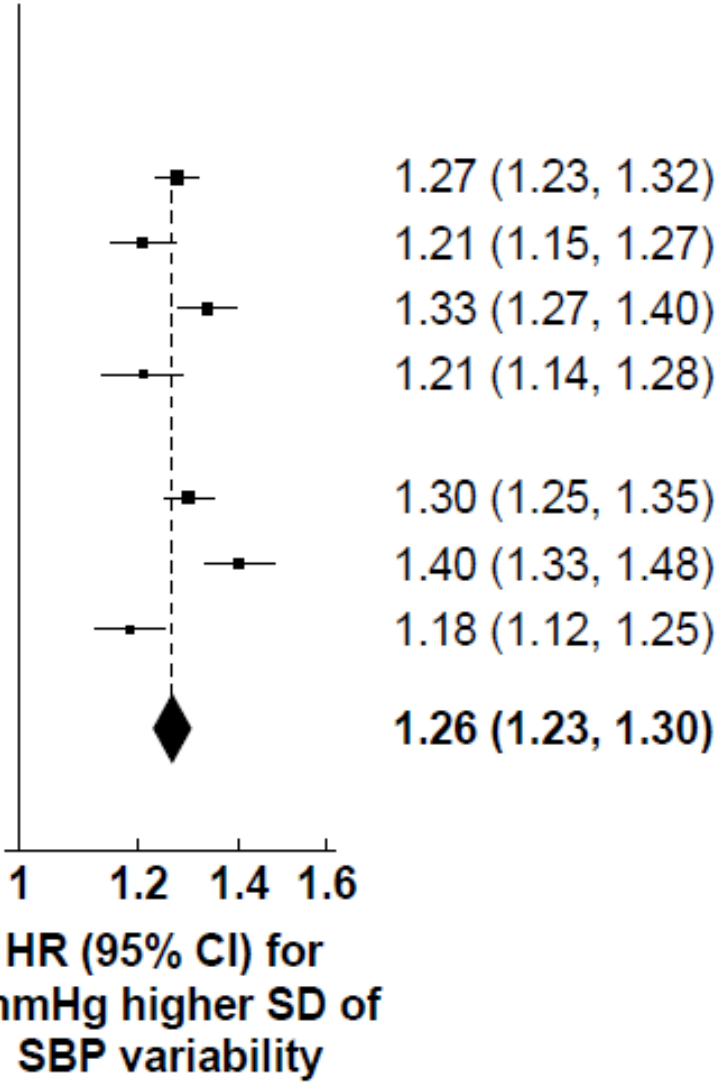

\section{Figure 4}

Adjusted hazard ratios for the risk of CVD, coronary heart disease, stroke, heart failure, all cause mortality, CVD mortality, non-CVD mortality and their composite with each $5 \mathrm{mmHg}$ increasing SBP variability using multivariable Cox regressions. HR was adjusted by age at risk, gender, smoking status, body mass index, SBP, diastolic blood pressure, haemoglobin A1c, low-density lipoprotein-cholesterol, estimated glomerular filtration rate, the usages of oral anti-diabetic drugs, insulin, angiotensin converting enzyme inhibitor/angiotensin receptor blocker, $\beta$-blocker, calcium channel blocker, diuretic, other anti-hypertensive drugs, lipid-lowering agent, Charlson's index and usual SBP. SD=Standard deviation; SBP=Systolic blood pressure; $\mathrm{CVD}=$ Cardiovascular disease; $\mathrm{HR}=$ Hazard ratio. 


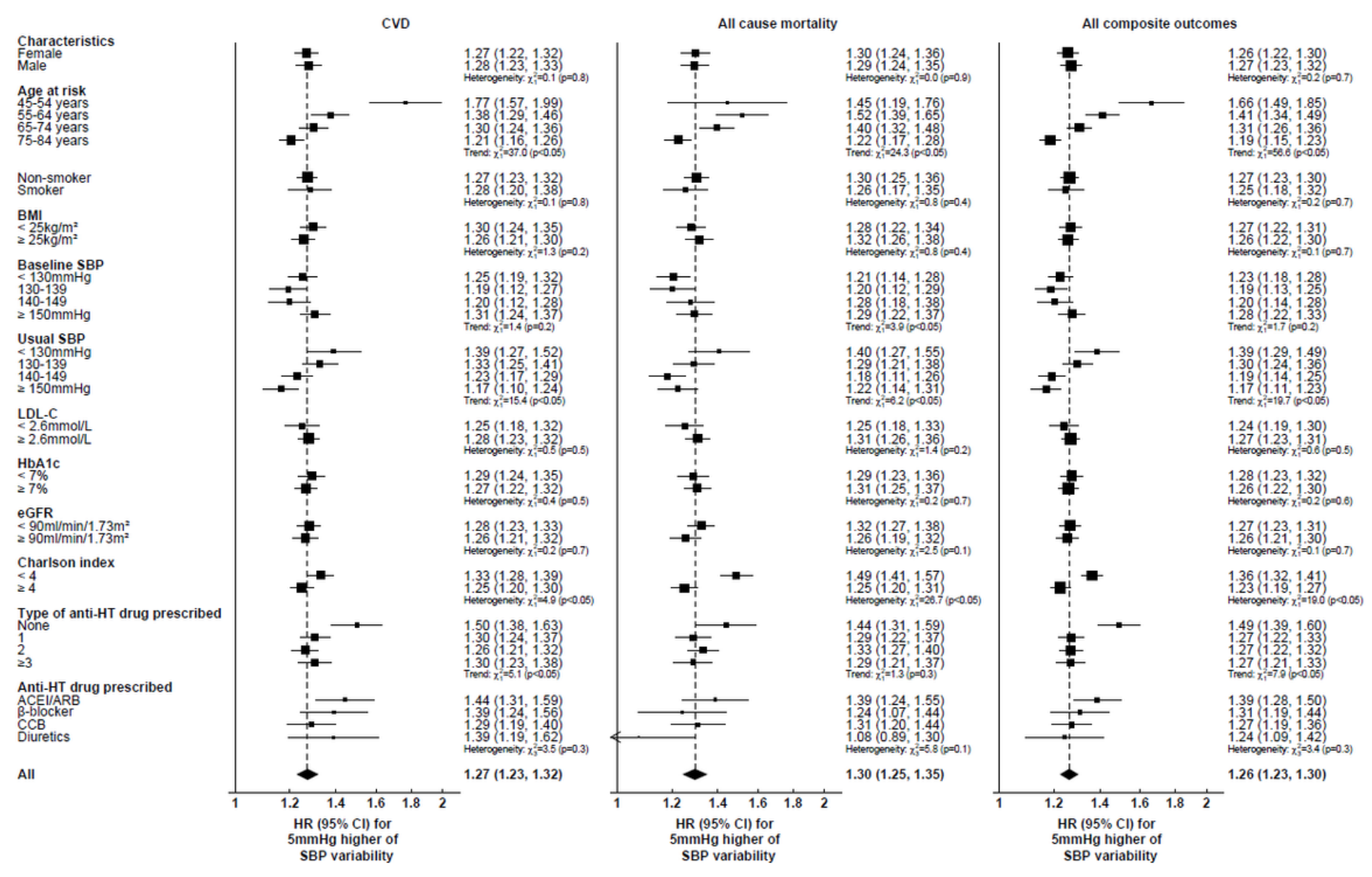

Figure 6

Adjusted hazard ratios for the risk of CVD, all cause mortality and their composite events with each $5 \mathrm{mmHg}$ increasing SBP variability by stratifying patient's characteristics at baseline using multivariable Cox regressions. HR was adjusted by age at risk, gender, smoking status, BMI, SBP, diastolic blood pressure, $\mathrm{HbA1c}$, LDL-C, estimated glomerular filtration rate, the usages of oral anti-diabetic drugs, insulin, angiotensin converting enzyme inhibitor/angiotensin receptor blocker, $\beta$-blocker, calcium channel blocker, diuretic, other anti-hypertensive drugs, lipid-lowering agent, Charlson's index and usual SBP. SBP=Systolic

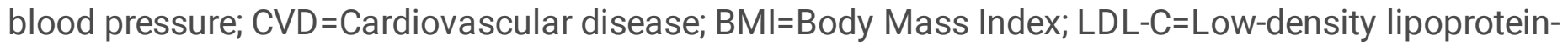
cholesterol; $\mathrm{HbA} 1 \mathrm{c}=$ Haemoglobin A1c; $\mathrm{HR}=$ Hazard ratio; eGFR=estimated glomerular filtration rate; $\mathrm{HT}=$ Hypertensive; $\mathrm{ACEI}=$ Angiotensin Converting Enzyme Inhibitor; $\mathrm{ARB}$ = Angiotensin Receptor Blocker; $\mathrm{CCB}=$ Calcium Channel Blocker; $\mathrm{Cl}=$ Confidence interval.

\section{Supplementary Files}

This is a list of supplementary files associated with this preprint. Click to download.

- Supplementarytablesfigures20200303.pdf

- Supplementarytablesfigures20200303.pdf 